\title{
A molecular signal for preeclampsia
}

The machine learning classifier was able to distinguish between pregnancies with early-onset preeclampsia and uncomplicated pregnancies

Preeclampsia is a common and serious complication of pregnancy and is associated with considerable global maternal and perinatal morbidity and mortality. A need exists for specific and sensitive, non-invasive tests for preeclampsia, to identify pregnant women at risk before the development of organ damage. New research published in Science Translational Medicine identifies a circulating RNA (C-RNA) signature that can classify pregnancies affected by preeclampsia.

Preeclampsia is currently diagnosed as maternal new-onset hypertension occurring after 20 weeks of gestation. "A pregnancy complication such as preeclampsia has a placental component, a fetal component and a maternal component (that is, the fetal and maternal responses to placental dysfunction)," explains corresponding author Fiona Kaper. "As C-RNA captures RNA from the placenta, maternal organs and potentially the fetus, we hypothesized that it could provide insight into pregnancy progression at a molecular level and potentially identify states associated with an adverse pregnancy event."

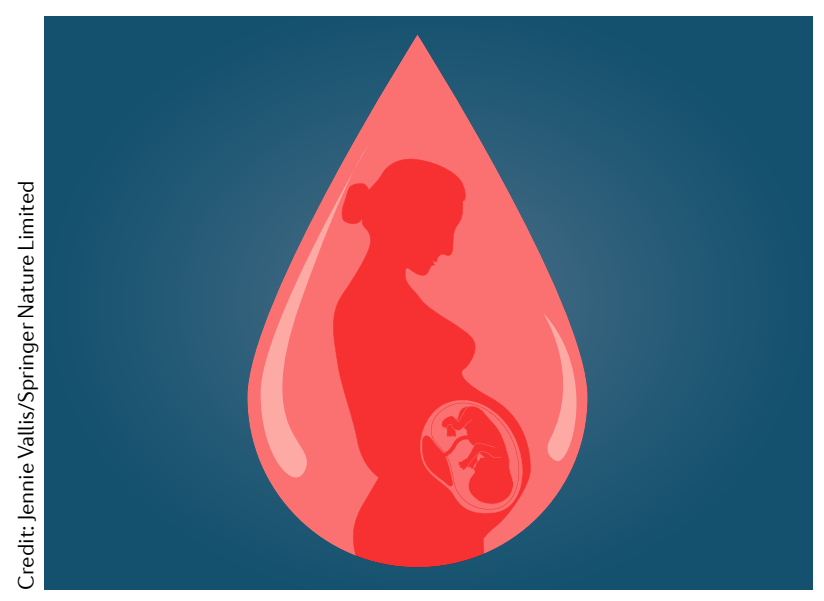

First, the researchers established a workflow to reproducibly assess C-RNA in plasma, which is present at low abundance. "To cast a wide net, we opted to take an agnostic approach; that is, rather than interrogating specific transcripts of placental origin, we aimed to assess the entire circulating transcriptome using RNA-sequencing methods," says Kaper. The most abundant transcripts present in human blood are ribosomal and globulin RNAs, which had to be depleted from plasma. Rather than use standard depletion methods, the researchers generated a library from all C-RNA and carried out probe-assisted enrichment targeting the whole human exome.

Using this method, C-RNA dynamics were tracked in 41 healthy pregnancies, from the first to the third trimester. Importantly, 156 differentially abundant transcripts were identified, the majority of which increased in abundance as pregnancy progressed. "We could follow the progression of pregnancy in a non-complicated cohort by temporal changes in transcript abundance throughout the course of healthy pregnancies," describes Kaper.

The new C-RNA approach was applied to two independent preeclampsia cohorts, iPEC and PEARL PEC. In the iPEC cohort, samples were collected from 40 women with severe early-onset preeclampsia at the time of diagnosis and from 73 control participants, matched for gestational age. The PEARL PEC cohort collected samples from 12 women with early-onset preeclampsia and 12 women with late-onset preeclampsia as well as 24 control participants matched for gestational age. "When we interrogated pregnancies affected by early-onset severe preeclampsia, we identified 30 transcripts in circulation that were differentially abundant compared with non-complicated pregnancies," says Kaper. "These transcripts had gene ontology annotations and tissue expression patterns consistent with the placental dysfunction, impaired fetal development, and maternal immune and cardiovascular dysregulation characteristic of preeclampsia."

To test whether the C-RNA changes detected in preeclampsia pregnancies could be used to classify preeclampsia, the researchers developed a machine learning classifier that used an Adaboost algorithm. Ten percent of the iPEC samples and all PEARL PEC samples were excluded from the model building, in order to assess performance. The machine learning classifier was able to distinguish between pregnancies with early-onset preeclampsia and uncomplicated pregnancies with $\geq 85 \%$ accuracy, and to identify late-onset preeclampsia with $72 \%$ accuracy.

"Future studies should focus earlier in pregnancy to evaluate the potential of this approach to improve prognostication and prediction of outcomes for women with preeclampsia and other pregnancy complications," concludes Kaper. "Such studies hold great promise for uncovering predictive biomarkers for early stratification of all at-risk pregnancies, informing prophylactic interventions, or more vigilant monitoring of pregnancy."

Shimona Starling

ORIGINAL ARTICLE Munchel, S. et al. Circulating transcripts in maternal blood reflect a molecular signature of early-onset preeclampsia. Sci. Transl Med. 12, eaaz0131 (2020) RELATED ARTICLE Aplin, J. D. et al. Tracking placental development in health and disease. Nat. Rev. Endocrinol. https://doi.org/10.1038/ s41574-020-0372-6(2020) 